\title{
Understanding the Benefits of Dynamic Line Rating under Multiple Sources of Uncertainty
}

\author{
Fei Teng, Member, IEEE, Romain Dupin, Andrea Michiorri, George Kariniotakis, Senior Member, IEEE, Yanfei
} Chen and Goran Strbac, Member, IEEE

\begin{abstract}
This paper analyses the benefits of dynamic line rating (DLR) in the system with high penetration of wind generation. A probabilistic forecasting model for the line ratings is incorporated into a two-stage stochastic optimization model. The scheduling model, for the first time, considers the uncertainty associated with wind generation, line ratings and line outages to co-optimize the energy production and reserve holding levels in the scheduling stage as well as the re-dispatch actions in the real-time operation stage. Therefore, the benefits of higher utilization of line capacity can be explicitly balanced against the costs of increased holding and utilization of reserve services due to the forecasting error. The computational burden driven by the modelling of multiple sources of uncertainty is tackled by applying an efficient filtering approach. The case studies demonstrate the benefits of DLR in supporting costeffective integration of high penetration of wind generation into the existing network. We also highlight the importance of simultaneously considering the multiple sources of uncertainty in understanding the benefits of DLR. Furthermore, this paper analyses the impact of different operational strategies, the coordination among multiple flexible technologies and installed capacity of wind generation on the benefits of DLR.

Index Terms-Dynamic line rating, probabilistic forecasting, stochastic programming, wind generation.
\end{abstract}

\section{NOMENCLATURE}

\section{A. Constants (Written in Normal Font)}

$\mathrm{D}_{n} \quad$ Demand in node $n(\mathrm{MW})$

$\underline{\mathrm{F}}_{l} / \overline{\mathrm{F}}_{l} \quad$ Minimum / Maximum capacity of AC transmission line $l(\mathrm{MW})$

$\overline{\mathrm{H}}_{g}^{u p} / \overline{\mathrm{H}}_{g}^{d o} \quad$ Maximum reserve up / down holding amount of conventional generator $g(\mathrm{MW})$

$\underline{\mathrm{P}}_{g} / \overline{\mathrm{P}}_{g} \quad$ Minimum / Maximum capacity of conventional generator $g(\mathrm{MW})$

$\overline{\mathrm{WP}}_{s, w} \quad$ Maximum available output of wind generator $w$ in scenario $s$ (MW)

$\mathrm{X}_{l} \quad$ Reactance of AC line $l$ (p.u.)

Voll $_{n} \quad$ Value of lost load at node $\mathrm{n}[\mathfrak{f} / \mathrm{MWh}]$.

F. Teng, YF. Chen and G. Strbac are with Department of Electrical and Electronic Engineering, Imperial College London, London, SW7 2AZ, U.K. (email: f.teng@imperial.ac.uk, yanfei.chen11@imperial.ac.uk, g.strbac (imperial.ac.uk.)

R. Dupin, A. Michiorri, G. Kariniotakis are with with MINES ParisTech, PSL Research University, PERSEE - Centre for Processes, Renewable Energies and Energy Systems CS 10207 rue Claude Daunesse, 06904 Sophia Antipolis Cedex, France. (email: romain.dupin@mines-paristech.fr, andrea.michiorri@mines-paristech.fr, georges.kariniotakis@mines-paristech fr)

$$
\begin{aligned}
& \pi \text { fix }_{g} \quad \text { Fixed/Commitment cost of conventional } \\
& \text { generator } g(£ / \mathrm{MW} / \mathrm{h}) \\
& \pi \text { fuel }_{g} \quad \text { Fuel cost of conventional generator } g(£ / \mathrm{MWh}) \\
& \pi r \quad \text { Utilization cost of reserve down service from } \\
& \text { conventional generator } g(£ / \mathrm{MWh}) \\
& \operatorname{Trup}_{g} \quad \text { Utilization cost of reserve up service from } \\
& \text { conventional generator } g(£ / \mathrm{MWh})
\end{aligned}
$$

B. Variables (Written in Italic Font)

$f_{s, l} \quad$ Power flow of AC line $l$ at scenario $s$ (MW)

$I_{g} \quad$ Binary variable. 1 means conventional generator $g$ is committed

$l l_{s, n} \quad$ Loss of load in node $k$ at operating state $s$ [MW]

$P_{g} \quad$ Output of conventional generator $g$ (MW)

$R_{s, g}^{u p} / R_{s, g}^{d o} \quad$ Utilized reserve up /down service from conventional generator $g$ at scenario s (MW)

$W P_{s, w} \quad$ Output of wind generator $w$ at scenario $s$ (MW)

$\Delta_{s, l} \quad$ Ratio of DLR to SLR for line $l$ at scenario s (p.u.)

$\theta_{s, n} \quad$ Voltage angle of node $n$ at scenario $s$ (rad)

\section{Set Related Constants (Written in Italic Font)}

$G_{n} \quad$ Set of conventional generators in node $n$

$I_{n} \quad$ Set of lines with positive power flow to node $n$

$n_{1}(l) \quad$ First end node of line $l$

$n_{2}(l) \quad$ Second end node of line $l$

$\mathrm{Ng} \quad$ Total number of conventional generators

$N l \quad$ Total number of transmission lines

Nn Total number of nodes

Ns Total number of scenarios

Nw Total number of wind generators

$O_{n} \quad$ Set of lines with positive power flow from node $n$

$W_{n} \quad$ Set of wind generators in node $n$.

\section{INTRODUCTION}

High penetration of renewable energy resources (RES) are expected to be integrated into the future power systems. However, there are two main challenges associated with such massive integration. Firstly, most of RES are intermittent, leading to an increased need on the ancillary services [1]. Novel stochastic operation frameworks, along with flexible technologies (such as energy storage), have been proposed to accommodate the intermittence of RES. Secondly, RES is usually located in the remote areas, and hence large-scale 
network expansion is required to transfer the low-carbon electricity to the load centre [2]. This not only requires large amount of investment, but also takes long time to develop, potentially limiting the penetration of RES in the short term.

Dynamic line rating (DLR) [3] is an alternative technology to enhance the transfer capability of the existing network. Traditionally, static line ratings (SLR) are utilized, while the actual line capacity depends on various factors that change in time, such as solar radiation and wind condition [4] SLR causes significant under-utilization of the actual network transfer capability. The application of DLR can effectively alleviate network congestion, lower the operating costs and RES curtailment in the short term and thus postpone and reduce the need of network reinforcement and support the high penetration of RES in the long term. Therefore, it is important to understand how DLR can be optimally integrated into the system operation and to what extent it can facilitate the cost-effective integration of RES [5].

The authors in [6] propose a general DLR calculation model and carry out cost-benefit analysis for the application of DLR. An "N-1" security constrained dispatch model is presented in [7] with the consideration of DLR and the results suggest that DLR reduces RES curtailment and dispatch cost with the expense of higher computational time for the scheduling problem. The study in [8] shows that the implementation of DLR to a $132 \mathrm{kV}$ line between Skegness and Boston can enable $20 \%$ to $50 \%$ more wind generation to be connected. The authors in [9] propose a distribution network investment model with DLR option to demonstrate the role of DLR in supporting distributed energy resources. Combined analysis of DLR and demand response in [10] identifies the synergy between these technologies. The authors in [11] apply a security constrained AC dispatch model to investigate the benefit of DLR in enhancing the system security.

However, like other weather-dependent elements, the line ratings cannot be perfectly forecasted in advance, which is not explicitly considered in the above literatures. The authors in [12] highlight the need to develop DLR forecast models to facilitate its application and present a state-of-art review on the forecasting techniques. Machine learning techniques [13] and ensemble weather forecast [14] are among the most widely used method in the forecasting of DLR. A novel probabilistic DLR forecasting method is developed in [15] to derive the expected value and important percentiles of ratings.

The forecasting error of DLR imposes significant challenges on its optimal implementation in the system operation. A cost-benefit based optimal quantile selection approach is developed in [23] and the selected quantile can be used as the input into the system scheduling model. The authors in [16] propose a novel robust congestion management model to optimally use DLR on critical lines, while keeping the risk of overloading below certain level. However, this model relies on a simple binary line rating forecast and requires pre-selected risk level. The work in [17] incorporates DLR with forecasting error distribution into a robust system dispatch framework, but only considers the uncertainty related to DLR forecast. Two-stage stochastic programming is the most widely used method to coordinate day-ahead dispatch and real-time re-dispatch actions under uncertainty [18]. However, none of existing stochastic model directly incorporates DLR into the optimal system operation when simultaneously considering forecasting errors of DLR and wind generation as well as line outages. Furthermore, in addition to DLR, there are multiple flexible network technologies (e.g. FACTs). In the literature and industrial applications, there is lack of understanding on how DLR can be optimally utilized to support high penetration of RES when considering the interaction among multiple sources of uncertainty and the coordination among multiple flexible technologies.

In this context, this paper extends a two-stage stochastic optimization model to include the probabilistic forecasts of DLR so that the model can co-optimize the energy and reserve holding levels in the scheduling stage as well as the re-dispatch actions in the real-time operation stage. Therefore, the benefit of higher utilization of line capacity can be explicitly balanced against the cost of increased holding and utilization of reserve services and/or demand shedding. The key contributions can be summarised as:

1) this paper presents a stochastic assessment framework with the probabilistic forecasts of DLR, which, for the first time, enables the understanding of optimal utilization of DLR with consideration of the interaction among multiple sources of uncertainty (DLR, wind outputs and line outages) and the coordination among multiple flexible network technologies (DLR and FACTs).

2) to tackle the computational burden driven by the large number of integer variables associated with FACTs devices and the large number of scenarios driven by the multiple sources of uncertainty, we investigate the minimal value of "big-M" used in the disjunctive approach and apply an iterative filtering model to select a subset of the scenarios to represent the multiple sources of uncertainty.

3) comprehensive analysis is carried out to understand the benefits of DLR in the system with high penetration of wind generation under different operational strategies and to investigate how the correlations among multiple sources of uncertainty as well as the coordination with FACTs devices may affect the benefits of DLR.

The paper is organized as follows. Section II presents the modelling framework for DLR and its forecasting. Section III introduces the two-stage stochastic scheduling model that is used to optimize the system operation with probabilistic forecasts of DLR. The benefits of DLR are firstly demonstrated through a simple 2-busbar system in Section IV and a study on IEEE 24 bus RTS system is then presented in Section V. Final conclusions are discussed in section VI.

\section{DYNAMIC LINE RATING}

\section{A. Modelling of Dynamic Line Rating}

The current carrying capacity of overhead line conductors is determined by the maximum sag, which guarantees the minimum allowed clearance. Conductors lose tensile strength and hence increase their sag with the increased temperature, 
as summarised in (1) with the link between tensile strength $F t$ and vertical sage Lsag and (2) with the relation between conductor temperature $T c$ and axial tensile strength $\mathrm{Ft}$.

$$
\begin{gathered}
L_{s a g} \approx \frac{F_{p} L_{\text {span }}^{2}}{8 F_{t}} \\
E_{c} A_{c} \beta_{c} T_{c, i}+\frac{E_{c} A_{c}}{24}\left(\frac{F_{p, i} L_{s p a n}}{F_{t, i}}\right)^{2}-F_{t, i}=K
\end{gathered}
$$

where $L_{s a g}[\mathrm{~m}]$ is sag, $F_{p}[\mathrm{~N}]$ is linear weight of the conductor, $F_{t}[\mathrm{~N}]$ is tensile strength of the conductor, $L_{\text {span }}[\mathrm{m}]$ is length of the span, $E_{c}[\mathrm{~Pa}]$ is Young's modulus, $A_{c}\left[\mathrm{~m}^{2}\right]$ is conductor cross sectional area, $\beta_{c}\left[\mathrm{~K}^{-1}\right]$ is conductor's expansion factor, $T_{c}[\mathrm{~K}]$ is conductor's temperature and $K[\mathrm{~N}]$ is a constant value.

This shows that the sag can be limited by constraining the conductor's temperature Tc. In the steady state, this parameter in turn is dependent on the thermal equilibrium between the heat dissipated by joule effect in the conductor and the heat exchange on its surface, as shown in Equation (3), usually represented in the form (4).

$$
\begin{aligned}
& q_{c}+q_{r}=q_{s}+q_{l} \\
& I=\sqrt{\frac{q_{c}+q_{r}-q_{s}}{R}}
\end{aligned}
$$

where $I[\mathrm{~A}]$ is maximum allowable current, $q_{c}[\mathrm{~W} / \mathrm{m}]$ is convective cooling, $q_{r}[\mathrm{~W} / \mathrm{m}]$ is radiative cooling, $q_{s}[\mathrm{~W} / \mathrm{m}]$ is solar heating and $\mathrm{R}[\Omega / \mathrm{m}]$ is conductor resistance.

As seen above, these terms depend on conductor's temperature and environmental parameters such as air temperature, wind speed and solar radiation. Details on the calculation on each one of these terms can be found in [6]. Due to the non-linear relations between conductor rating and wind speed and the variability of environmental parameters, it is common practice to use static ratings based on conservative assumptions. This has two main drawbacks: the use of a small part of the circuits' current carrying capacity and the presence of high risk situations where real ratings are below the SLR.

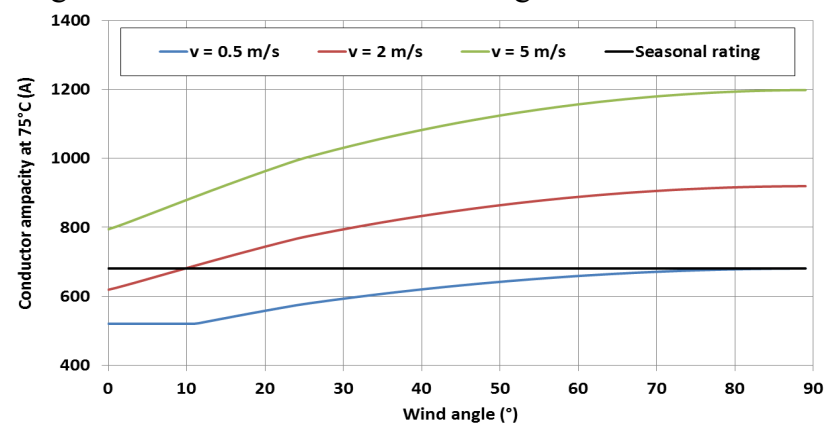

Fig.1 Relation among wind speed, wind direction and conductor ampacity

For an example of a Lynx cable with a maximum temperature of $75^{\circ} \mathrm{C}$ and according to the CIGRE standard, Fig.1 shows an illustration of the relationship between conductor rating, wind speed and wind direction, the ambient temperature being set to $20^{\circ} \mathrm{C}$ and the global solar radiation being null. These are compared with the summer static seasonal rating. The static seasonal rating is the current standard adopted by network operators for setting line maximum ampacity and is calculated considering static values for weather parameters. In this case the values used are: $0.5[\mathrm{~m} / \mathrm{s}]$ for wind speed, wind direction perpendicular to the axis of the conductor, $20\left[{ }^{\circ} \mathrm{C}\right]$ for air temperature and 0 $\left[\mathrm{W} / \mathrm{m}^{2}\right]$ for solar radiation.

\section{B. Forecast for Dynamic Line Rating}

Like other weather-dependent elements, the actual line ratings cannot be perfectly forecasted in advance, which explains the slow take-up rate of this technology. Due to the strong dependence of DLR on the weather conditions, the procedure proposed for DLR forecasts is similar to the approach commonly used for renewable energy production forecast, i.e. based on a mix of statistical methods and meteorological forecasts. The problem of forecasting is treated as a regression problem, where it is to be found a function $f_{h}$ able to provide an ampacity forecast $\hat{Y}_{t+h \mid t}$ at time $t+h$ knowing a set of information $X_{t}$ at time $t$.

The line rating forecast $\hat{Y}_{t+h \mid t}$ at a time $t$ for a horizon $t$, considering a series of explanatory variables $X_{t}$ is given by:

$$
\widehat{Y}_{t+h \mid t}=f_{h}\left(X_{t}\right)
$$

where $f_{h}$ returns the random variable $Y_{t+h}$ with an explicit distribution. Given that the conductor ampacity is not observable, we consider it as a nonlinear function $g($.$) of$ exogenous variable $X_{t}$. This function does not depend on the time step and can be calculated as detailed in $[4,6]$.

System operators consider security constraints in a conservative way, and so the probability of having a forecast higher than the observation must be set as equal to a value $\tau$ defined by the operator as specified in (6). Such forecasts can be provided through various methods, directly providing a quantile forecast $\widehat{Y}_{\mathrm{t}+\mathrm{h} \mid \mathrm{t}}^{\tau}$ or a probability density function $\hat{\mathrm{f}}_{\mathrm{h}}$ used for its calculation.

$$
P\left(\hat{Y}_{t+h \mid t}>Y_{t+h}\right)=\tau
$$

In this work, DLR forecasts are calculated with a Quantile Regression Forest algorithm [19], a machine learning method successfully used for probabilistic ampacity forecasts [13]. In this method, $\mathrm{k}$ different decision trees are generated and trained on a randomly generated dataset through bootstrap aggregation. After this at each split of the tree, features are selected through a random features' subset. The model calculates the $\mathrm{k}$ different ampacity values describing a distribution of results from what is possible to calculate an average or the values of different quantiles.

An example of the forecasts is presented in Fig.2. Historical records for wind and other weather parameters relevant for the calculation of DLR relative to southern Sweden for the year 2010 have been used. The records contain hourly measurements of wind speed, wind direction, solar radiation and air temperature. They are coupled with hourly Numerical Weather Prediction for horizon up to $48 \mathrm{~h}$ for the same variables and updated once a day. These forecasts are obtained from the European Centre for MediumRange Weather Forecasts. 


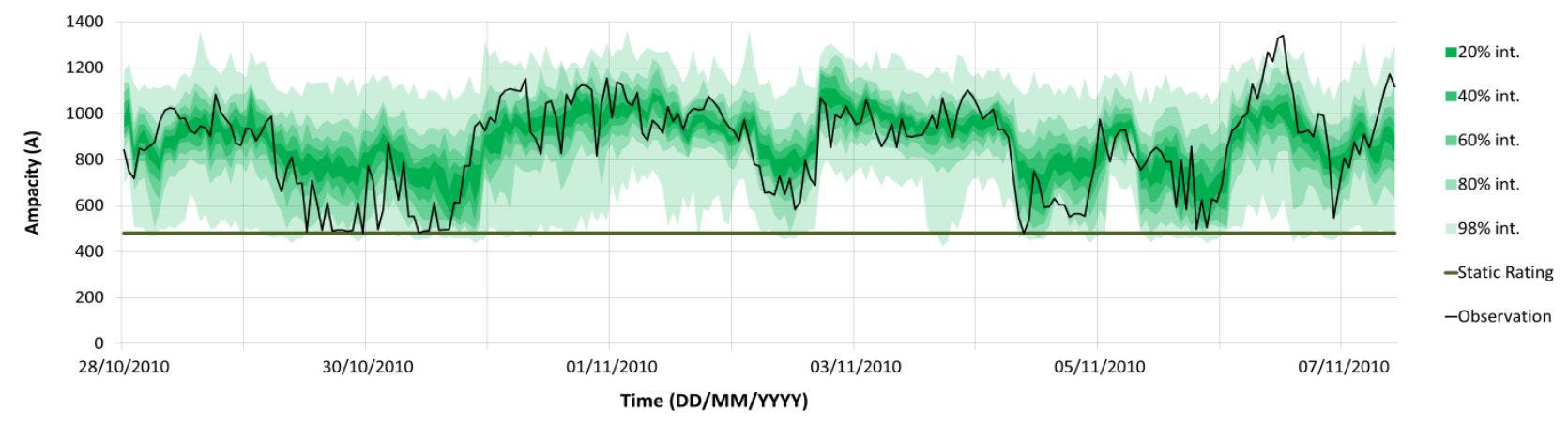

Fig. 2: Example of Dynamic Line Rating observations and day-ahead probabilistic forecasts

\section{Assessment ApproACH: TwO-STAGE StOCHAstic OPTIMIZATION MODEL}

A two-stage stochastic optimization model is proposed based on the scenarios that combine the possible realizations of line ratings, wind levels and line outages. The corrective cost-benefit based framework incorporates control actions from all sources (generators, demand and network) during pre and post-fault conditions. The aim of this framework is to efficiently balance costs of generation dispatch (including energy supply and reserve holding) and costs of corrective control actions (including reserve utilization and post-fault demand shedding).

The objective function of the probabilistic optimization is introduced in (7), where " $t$ " is the time duration of each selected operating condition and " $\rho_{s}$ " represents the probability of the selected scenario " $s$ ". The model is formulated as a mixed integer linear programming problem. The first components represent the dispatch costs (variable and fixed/commitment cost of conventional generators) in the scheduling stage, and the second and third cost components represent the costs of corrective control actions from generation side (reserve up/down services activation) and demand side (post-fault demand shedding) in each of the potential realizations.

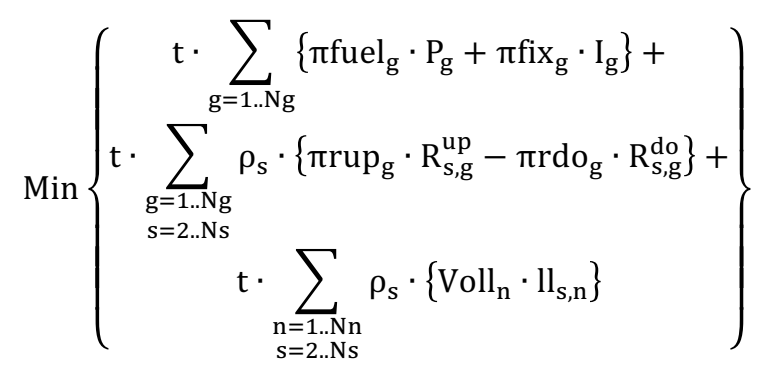

The optimization problem is subject to the following constraints. Wind generator output (8) is a decision variable through each node of the scenario tree. Generation capacity boundary constraint is formulated in (9). The availability of reserve provision is constrained by the commitment decisions and physical limits of conventional generation, as in (10)-(16). Power flows in the network are calculated in (17)-(18), while power balance constraint containing wind generator for each node is given in (19).

$$
\begin{array}{rr}
0 \leq W P_{s, g} \leq \overline{W P}_{s, g} & \forall s=2 . . N s \quad \forall g=1 . . N w \\
P_{g} \cdot I_{g} \leq P_{g} \leq \bar{P}_{g} \cdot I_{g} & \forall g=1 . . N g
\end{array}
$$

$$
\begin{aligned}
& H_{g}^{u p} \leq \bar{H}_{g}^{u p} \cdot I_{g} \\
& H_{g}^{d o} \leq \bar{H}_{g}^{d o} \cdot I_{g} \\
& \forall g=1 . . N g \\
& H_{g}^{u p} \leq \bar{P}_{g}-P_{g} \\
& \forall g=1 . . N g \\
& \forall g=1 . . N g \\
& H_{g}^{d o} \leq P_{g}-P_{g} \\
& \forall g=1 . . N g \\
& 0 \leq R_{s, g}^{u p} \leq H_{g}^{u p} \\
& 0 \leq R_{s, g}^{d o} \leq H_{g}^{d o} \\
& R_{s=1, g}^{u p}=R_{s=1, g}^{d o}=0 \\
& f_{s, l}=\frac{\theta_{s, n_{1}(l)}-\theta_{s, n_{2}(l)}}{X_{l}} \\
& \forall s=2 . . N s, g=1 . . N g \\
& \forall s=2 . . N s, g=1 . . N g \\
& s=1, \forall g=1 . . N g \\
& \forall s, l=1 . . N l \\
& \theta_{s, n=r e f}=0 \\
& \sum_{g \in G_{n}}\left(P_{g}+R_{s, g}^{u p}-R_{s, g}^{d o}\right)+\sum_{w \in W_{n}} W P_{s, g}+\sum_{l \in I_{n}}\left(f_{s, l}+P_{s, l}^{S C}\right) \\
& -\sum_{l \in O_{n}}\left(f_{s, l}+P_{s, l}^{S C}\right) \\
& =D_{n}-l l_{s, n} \\
& \forall s, n=1 . . N n
\end{aligned}
$$

Lines without and with DLR are contained in set A and B, respectively. The traditional line rating constraint is introduced in (20), while the constraint (21) is utilized to represent the lines equipped with DLR. In the case of line outage, constraint (22) is imposed.

$$
\begin{array}{lc}
F_{l} \leq f_{s, l}+P_{s, l}^{S C} \leq \bar{F}_{l} & \forall s, l \text { available, } l \in A \\
F_{l} \cdot \Delta_{s, l} \leq f_{s, l}+P_{s, l}^{S C} \leq \bar{F}_{l} \cdot \Delta_{s, l} & \forall s, l \text { available }, l \in B \\
f_{s, l}+P_{s, l}^{S C}=0 & \forall s, l \text { outage }
\end{array}
$$

With the disjunctive approach, power flow modified by series compensation (SC) can be determined by:

If $\Delta \theta_{\mathrm{s}, \mathrm{l}}$ is positive, then

$$
\varphi_{l} \cdot \Delta \theta_{s, l} \leq P_{s, l}^{S C} \leq \eta_{l} \cdot \Delta \theta_{s, l} \quad \forall s, l \in L
$$

If $\Delta \theta_{\mathrm{s}, \mathrm{l}}$ is is negative, then

$$
\eta_{l} \cdot \Delta \theta_{s, l} \leq P_{s, l}^{S C} \leq \varphi_{l} \cdot \Delta \theta_{s, l} \quad \forall s, l \in L
$$

To linearize this, "big-M" approach is applied to transfer (23)(24) into 4 mixed integer linear constraints (25)-(28):

$$
\begin{array}{ll}
P_{s, l}^{S C} \leq \eta_{l} \cdot \Delta \theta_{s, l}+Y_{s, l} \cdot M_{s, l} & \forall s, l \in L \\
P_{s, l}^{S C} \geq \varphi_{l} \cdot \Delta \theta_{s, l}-Y_{s, l} \cdot M_{s, l} & \forall s, l \in L \\
P_{s, l}^{S C} \geq \eta_{l} \cdot \Delta \theta_{s, l}-\left(1-Y_{s, l}\right) \cdot M_{s, l} & \forall s, l \in L \\
P_{s, l}^{S C} \leq \varphi_{l} \cdot \Delta \theta_{s, l}+\left(1-Y_{s, l}\right) \cdot M_{s, l} & \forall s, l \in L
\end{array}
$$

where $\eta_{l}=\frac{\bar{X}_{l} S C}{X_{l} *\left(X_{l}-\bar{X}_{l}^{S C}\right)}, \varphi_{l}=\frac{\underline{X}_{l}^{S C}}{\left.X_{l^{*}\left(X_{l}-\underline{X}_{l}\right.}^{S C}\right)}, \Delta \theta_{s, l}=\left(\theta_{s, k_{1}(l)}-\right.$ $\left.\theta_{s, k_{2}(l)}\right)$ and $Y_{s, l}$ is a binary variable. 
The parameter $" M_{s, l} "$ is a sufficient large number. However, inappropriate selection of $M_{s, l}$ may lead to long calculation time and limit the application of the model. Hence a minimum value of $M_{s, l}$ is investigated to accelerate the optimization. When $\Delta \theta_{\mathrm{s}, 1}$ is positive and $Y_{s, l}$ is 0 , in order to guarantee only (25) and (26) are activated, the right-hand side (RHS) of (28) must be bigger than the RHS of (25), and the RHS of (27) must be smaller than the RHS of (26). Consequently, (29) can be deduced as

$$
\left(\eta_{l}-\psi_{l}\right) \cdot \Delta \theta_{s, l} \leq \mathrm{M}_{s, l} \quad\left(\Delta \theta_{s, l}>0\right) \quad \forall s, l \in L
$$

Similarly, when $\Delta \theta_{s, l}$ is negative and $\mathrm{Y}_{\mathrm{s}, \mathrm{l}}$ is 1 , in order to only activate (27) and (28), the following constraint needs to be met:

$$
\left(\psi_{l}-\eta_{l}\right) \cdot \Delta \theta_{s, l} \leq \mathrm{M}_{s, l} \quad\left(\Delta \theta_{s, l}<0\right) \quad \forall s, l \in L
$$

Combine (29) and (30), the range of $\mathrm{M}_{s, l}$ can be obtained:

$$
\left(\eta_{l}-\psi_{l}\right) \cdot\left|\Delta \theta_{s, l}\right| \leq \mathrm{M}_{s, l} \quad \forall s, l \in L
$$

Assume $\Delta \theta_{s, l}=f_{s, l} \cdot\left(\mathrm{X}_{l}-X_{s, l}^{S C}\right)$ is used to calculate the power flow over the line installed with SC. If $X_{s, l}^{S C}=\underline{X}_{l}^{S C}=-\delta \cdot \mathrm{X}_{l}$, where $\delta$ is the maximum SC degree of compensation, then the positive maximum value of $\Delta \theta_{s, l}$ is $\overline{\mathrm{f}}_{\mathrm{s}, l} \cdot(1-(-\delta)) \cdot \mathrm{X}_{l}$. Since $\left(\eta_{l}-\psi_{l}\right)>0$, the minimum $\mathrm{M}_{\mathrm{s}, l}$ is hence $\frac{2 \delta}{1-\delta} \cdot \bar{f}_{s, l}$.

In the system with large amount of DLR devices, significantly increased number of scenarios are required to represent the multiple sources of uncertainty. This leads to high computational burden and may potentially prevent the implementation of DLR in the large scale system. Consequently, an extended filtering model based on [21] is applied in this paper to select a subset of scenarios so that the balance between computational time and optimality can be achieved. The fundamental idea is to select the scenarios that have high impact on the objective function for a given dispatch and hence may lead to a change of dispatch decision.

The proposed modelling framework can be executed iteratively by the following steps:

1). Input the weather forecasts into the proposed DLR forecasting model and obtain the associated DLR forecasts;

2). Define the full set of scenarios through combining DLR forecasts, wind forecasts as well as line outages; the initial subset is also selected;

3). Run the two-stage stochastic optimization framework based on the selected subset;

4). Calculate the probability weighted costs for all the scenarios with respect to scheduling decisions from step (3); this step is carried out by multi-threating parallel processing; 5). Rank the scenarios that are not in the selected subset with a descending order based on the calculated costs.

6). If the total cost of the scenarios, that are not in the selected subset, is higher than the pre-set tolerance, select and add the first ' $m$ ' scenarios with highest costs into the subset, and go to step (3); or, the algorithm terminates;

FICO Xpress v7.8 [22] is used on a server with two Intel Xeon E5-2687W processors and $512 \mathrm{~GB}$ of RAM to implement this algorithm.

\section{SMAll-SCAlE System StUdy}

To demonstrate the impact of DLR on the system operation, the two-stage stochastic scheduling model is firstly applied in a simple 2-busbar system, where the line outage is also neglected. There are four conventional generators (G1-G4) located in node 1, two conventional generators (G5-G6) located in node 2 and a wind farm with capacity of $70 \mathrm{MW}$ located in node 2. The physical limits and cost information for the generators are summarised in TABLE I. In addition, 70 MW demand is located in the node 1 , while a transmission line with DLR of $26 \mathrm{MW}$ links these two nodes. The value of

\begin{tabular}{|c|c|c|c|c|c|c|}
\hline $\begin{array}{l}\stackrel{5}{0} \\
\frac{\pi}{4} \\
\stackrel{0}{0} \\
0\end{array}$ & 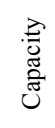 & 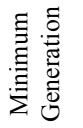 & 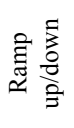 & $\underset{\text { II }}{\stackrel{\Phi}{\Xi}}$ & 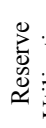 & $\begin{array}{l}\vec{d} \\
\stackrel{x}{\mid}\end{array}$ \\
\hline & \multicolumn{3}{|c|}{ MW } & \multicolumn{2}{|c|}{$£ / M W h$} & $£ / \mathrm{MW} / \mathrm{h}$ \\
\hline G1 & 20 & 7 & 13 & 70 & 70 & 600 \\
\hline G2 & 15 & 5 & 10 & 50 & 50 & 300 \\
\hline G3 & 15 & 5 & 10 & 50 & 50 & 300 \\
\hline G4 & 15 & 5 & 10 & 50 & 50 & 300 \\
\hline G5 & 5 & 1 & 3 & 30 & 30 & 400 \\
\hline G6 & 12 & 3 & 10 & 15 & 15 & 50 \\
\hline W1 & 70 & 0 & 0 & 0 & 0 & 0 \\
\hline
\end{tabular}
lost load is set to be $30 \mathrm{kf} / \mathrm{MWh}$.

TABLE I TWO-NODE SYSTEM'S GENERATION AND NETWORK DATA

This case study considers two sources of uncertainty, forecasting errors of DLR and wind generation. The expected wind production is assumed to $50 \%$ of the installed capacity and the forecasting error is assumed to follow a normal distribution with 0.2 standard deviation. 9 scenarios are generated to describe the distribution of wind forecasting. Table II presents a sample of the probabilistic forecasts of DLR. In the base case analysis, the two forecasting errors are assumed to be independent and hence there are in total 81 scenarios to be considered.

TABLE II DYNAMIC LINE RATING SCENARIOS

\begin{tabular}{|c|c|c|c|c|c|c|c|c|c|}
\hline $\begin{array}{c}\text { Rating over } \\
\text { SLR }\end{array}$ & 1 & 1.1 & 1.2 & 1.3 & 1.4 & 1.5 & 1.6 & 1.7 & 1.8 \\
\hline $\begin{array}{c}\text { Probability } \\
(\%)\end{array}$ & 0.5 & 4 & 12 & 15 & 40 & 20 & 5 & 3 & 0.5 \\
\hline
\end{tabular}

\section{A. Benefits of DLR}

To understand the benefits of DLR, three different cases are compared:

1) SLR: No DLR monitoring stations are deployed. Hence, SLR strategy is used in both scheduling and operation phases. This approach represents the counterfactual case, against which the benefits of DLR can be assessed.

2) Real-time only DLR: DLR monitoring stations are assumed to be installed, but the forecast of DLR is not available. Therefore, SLR is applied in the scheduling phase, while DLR is allowed by using the data from monitoring stations in the real-time operation.

3) Fully-optimized DLR: DLR monitoring stations are assumed to be installed and the forecast of DLR is also available. DLR is therefore adopted in both the scheduling and the real-time operation phases. 
The results in TABLE III show that the implementation of DLR in real-time only reduces the operation cost by 166 $\mathrm{K} £$ and wind curtailment by $6 \mathrm{MW}$, while under the fullyoptimized case, the operation cost saving of $315 \mathrm{~K} £$ and wind curtailment reduction of $6 \mathrm{MW}$ can be achieved.

The real-time only DLR case has the same dispatch decision as SLR case, but the extra available capacity from DLR in the real-time operation allows accommodating more wind energy to replace high cost generators in Node 1. The expected wind curtailment is reduced by three times. Negative re-dispatch cost in Real-time only DLR is achieved through utilizing reserve down services in real time. In this case, the expected utilization of transmission line is increased from 26 MW to 32.4 MW. However, the dispatch decision with SLR leaves the low-cost generators G6 off-line, which limits the further utilization of DLR in the real time even when extra capacity becomes available.

For the fully optimised case, the optimal flow over the transmission line in the dispatch stage is increased to 36.4 MW through balancing the cost and benefit associated with higher transfer capacity. The additional transfer capacity allows the low-cost generator G6 in Node 2 to stay online. In addition, the wind generation can be fully absorbed in the scheduling stage, leading to a significant cost reduction. However, it is worth to point out that the fully optimized DLR case increases the amount of the reserve holding and the expected demand shedding in order to better utilize the lowcost resources in Node 2. Compared with SLR where the wind penetration is limited to $38 \%$, DLR allows the wind penetration to reach above $46 \%$.

\begin{tabular}{|c|c|c|c|c|}
\hline \multicolumn{2}{|c|}{ Approach } & \multirow{2}{*}{$\begin{array}{l}\text { SLR } \\
2018\end{array}$} & \multirow{2}{*}{$\begin{array}{l}\begin{array}{l}\text { Real-time } \\
\text { only DLR }\end{array} \\
2018\end{array}$} & \multirow{2}{*}{$\begin{array}{l}\text { Fully- } \\
\text { optimized } \\
\text { DLR } \\
1661\end{array}$} \\
\hline \multirow{4}{*}{ 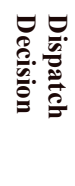 } & Dispatch Cost [£/30mins] & & & \\
\hline & Line power flow [MW] & 26 & 26 & 36.4 \\
\hline & Reserve holding [MW] & 26 & 26 & 32.4 \\
\hline & Wind curtailment [MW] & 9 & 9 & 0.6 \\
\hline \multirow{5}{*}{ 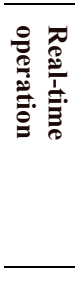 } & $\begin{array}{l}\text { Expected re-dispatch cost } \\
{[£ / 30 \text { mins }]}\end{array}$ & 9 & -158 & 41 \\
\hline & $\begin{array}{l}\text { Expected demand shedding } \\
\text { cost }[£ / 30 \mathrm{mins}]\end{array}$ & 4 & 4 & 10 \\
\hline & $\begin{array}{l}\text { Expected line power flow } \\
{[\mathrm{MW}]}\end{array}$ & 25.7 & 32.4 & 36 \\
\hline & $\begin{array}{l}\text { Expected wind curtailment } \\
{[\mathrm{MW}]}\end{array}$ & 9 & 3 & 3 \\
\hline & Total Cost $[£ / 30 \mathrm{mins}]$ & 2031 & 1865 & 1716 \\
\hline
\end{tabular}

\section{B. Reserve Sharing among Mutiple Sources of Uncertainty}

Previous work [17] investigates the optimal utilization of DLR under uncertainty, but only considers the forecasting error associated with DLR. The paper concludes that significant amount of reserve services are required to accommodate the forecasting error of DLR. However, there are multiple sources of uncertainty existing in the power system. They can in fact share some of reserve services and hence reduce the need of extra reserve services driven by each individual source of uncertainty.

By comparing the reserve levels under SLR and DLR in Table IV, it is clear that $26 \mathrm{MW}$ of extra reserve needs to be held if DLR is the only source of uncertainty, while if both forecasting errors of DLR and wind are considered, only 6.4 MW of extra reserve is needed. Similar conclusion can be drawn that with the optimal application of DLR, the reserve services driven by wind forecasting error itself reduces. TABLE IV RESERVE HOLDING LEVELS

\begin{tabular}{l|l|l}
\hline & SLR & DLR \\
\hline With Wind Forecasting Error (MW) & 26 & 32.4 \\
\hline Without Wind Forecasting Error (MW) & 0 & 26 \\
\hline
\end{tabular}

However, the reserve sharing effect between wind farm and DLR also depends on the locations of the sources of uncertainty. If the wind farm is located in the "importing" area, as opposed to the above case where the wind farm is located in the "exporting" area, the reserve sharing effect between DLR and wind farm is negligible.

\section{Correlation between Forecasting Errors}

In the previous analysis, the forecasting errors of wind generation and DLR are assumed to be independent. However, the positive correlation between DLR and near-by wind farm production has already been observed [20]. To demonstrate the impact, the correlation between forecasting errors of wind generation and DLR is assumed to be "1". According to the results in TABLE V, positive correlation increases the benefit of DLR by $16 \%$. This is due to the fact that the realization of higher wind availability in exporting area can be accommodated by the realization of higher line capacity from DLR when the correlation is positive.

TABLE V IMPACT OF CORRELATION ON THE BENEFITS OF DLR

\begin{tabular}{l|c|c}
\hline & Positively correlated & Independent \\
\hline Benefits [£/30mins] & 367 & 315 \\
\hline
\end{tabular}

\section{IEEE 24-BUS RTS SYSTEM}

To further understand the benefits of DLR, the proposed optimization framework is applied on a modified 24-bus IEEE RTS system, as shown in Fig.3. The specifications of generators, branches and demand can be found in [21]. Fuel costs of generation technologies are 7, 50, 125 and $8 £ / \mathrm{MWh}$ for nuclear, coal, oil and hydro, respectively. The reserve utilization costs are assumed to be same as fuel costs. The value of lost load is $30 \mathrm{kf} / \mathrm{MWh}$. To increase the need for extra transmission capability of the North-South boundary, the demand levels in node 1-3 are doubled and 200/500 MW of nuclear plants are added in node 22/23. Generators in node 18 and node 21 are replaced by $2600 \mathrm{MW}$ wind farm. Additional $1000 \mathrm{MW}$ wind farm is placed in node 16 . Two lines, marked with red colour in Fig.3, are equipped with DLR devices. The SLR of the lines are $350 \mathrm{MW}$. The forecasting errors of wind generation and DLR remain the same as in the 2-busbar system. In this case, by combining 9 scenarios for each of two lines with DLR, 9 scenarios for wind generation and 38 potential line outages, there are 27702 scenarios in total. The proposed filter is hence applied to accelerate the calculation.

\section{A. Impact of Operation Strategies on the Benefit of DLR}

Alternative operation strategies have been proposed for DLR. This section compares the benefits of DLR under three different strategies, the results of which can be used as a guideline for the optimal implementation of DLR. In the base case that SLR is applied, the operation cost is $42.3 \mathrm{k} £$ and 525 MWh of wind generation is curtailed. 


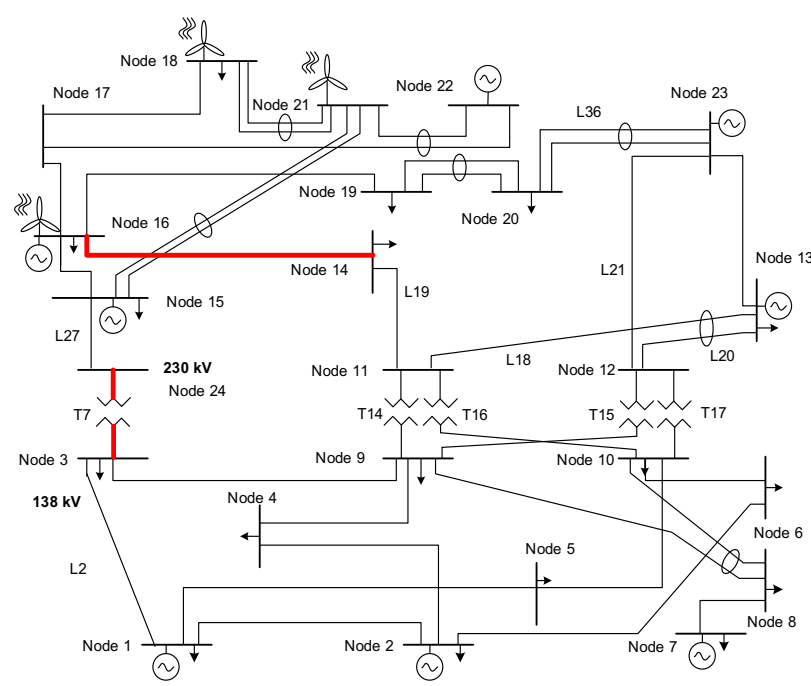

Fig. 3. Modified IEEE 24-Node RTS Topology

For the application of DLR, the simplest way is to implement it only during the real-time operation stage when the actual ratings of the lines become available. In this case, SLR is used in the dispatch stage and re-dispatch actions are activated in the real-time stage to make use of the extra transfer capability. In this case, the cost reduces to $35.1 \mathrm{k} £$ and only $387 \mathrm{MWh}$ of wind generation are curtailed.

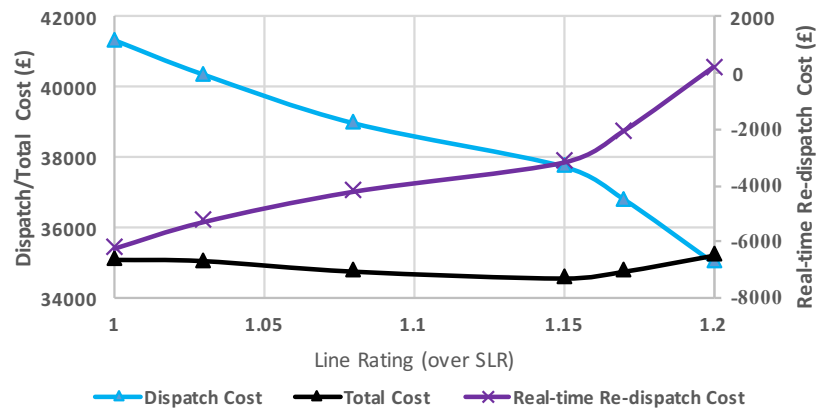

Fig. 4. Operation cost under different quantiles of DLR

Furthermore, previous study in [24] has been carried out to select the single "optimal" quantile of DLR forecast to be used in the dispatch stage under a deterministic fashion. As shown in Fig.4, when higher line rating is used in the dispatch stage, the system dispatch cost reduces due to the increased transfer capability, while the real-time re-dispatch cost would increase in order to deal with the forecasting error in the real-time operation. Therefore, the optimal balance between these two costs leads to the maximum benefit of DLR. In the given case, 1.15 over SLR is the optimal selection with the total operation cost at $34.5 \mathrm{kf}$ and wind curtailment at $428 \mathrm{MWh}$.

Instead of pre-selecting a single rating, it is possible to explicitly optimize the application of DLR by using the probabilistic forecasts of DLR in the proposed two-stage stochastic dispatch model. In this way, the benefits of higher utilization of line capacity can be explicitly balanced against the costs of increased holding and utilization of reserve services. The fully-optimized case leads to the lowest operation cost at $32.3 \mathrm{k} £$ and wind curtailment at $316 \mathrm{MWh}$.

The results clearly demonstrate that the optimal application of DLR can not only save the system operation cost but also reduce the wind curtailment to achieve higher penetration of wind generation in the existing network. However, it is worth noting that considering the multiple sources of uncertainty significantly increases the computational burden. For the case that SLR is applied, the computational time is $65 \mathrm{~s}$. For the fully-optimized case without filter, it takes more than 3 hours to solve the full problem and hence is not applicable in the large-scale system. By applying the proposed filter with multithreating parallel processing, the optimization is finished in $497 \mathrm{~s}$ with $0.5 \%$ tolerance.

\section{B. Coordination with FACTs Devices}

In addition to DLR, there are multiple flexible network technologies. There is lack of understanding on how DLR can be optimally coordinated with other flexible technologies and whether they would compete or facilitate with each other. FACTS devices are one of the most efficient and reliable solution to increase the flexibility of the network. In this section, we demonstrate the potential synergy between DLR and FACTs devices.

Two series compensation devices with \pm 0.4 degree of compensation capability are installed at line 2 and line 18. The results in Fig. 5 show that the benefit of DLR (cost difference between DLR and SLR) can be potentially increased by $30 \%$ through the optimal coordination with FACTs devices. Moreover, the result suggests that the extra benefits from coordinating with FACTs devices is mainly from reducing the cost to deal with the DLR forecasting error.

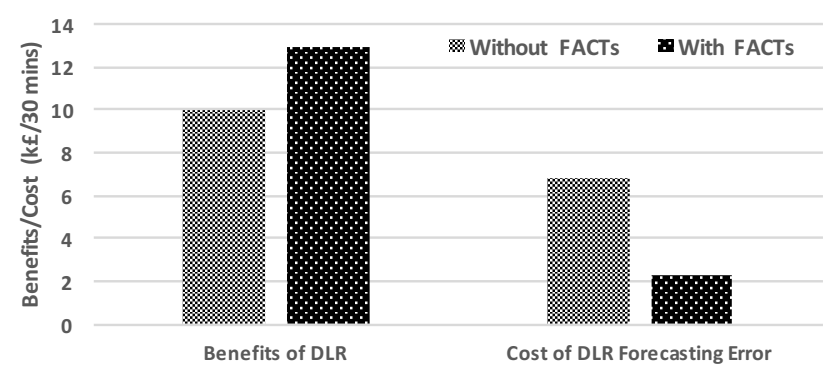

Fig. 5. Benefit of DLR with/without FACTs

\section{Impact of Installed Capacity of Wind Generation}

This section analyses the impact of different levels of installed wind generation on the benefit of DLR. The results in Fig. 6 clearly show that a higher installed capacity of wind generation leads to higher benefit of DLR. This is driven by the increased need to transfer the low cost wind generation through the North-South boundary. However, the results also demonstrate that after a certain level of installed capacity, the benefit of DLR tends to saturate, implying the need for extra transmission capability through alternative measures.

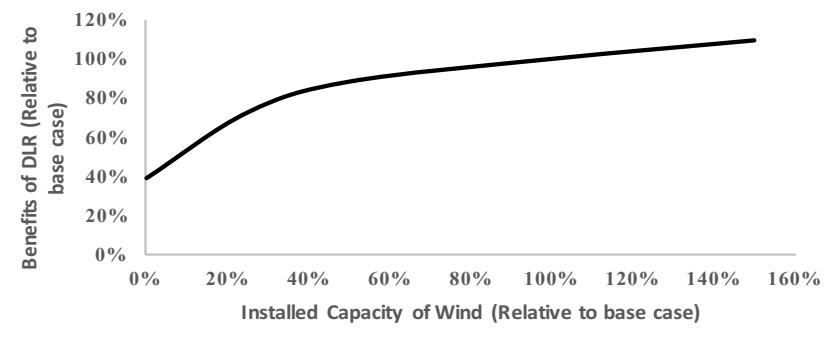

Fig. 6. Impact of installed wind capacity on the benefits of DLR 


\section{CONCLUSIONS AND FUTURE WORK}

This paper investigates the benefits of the optimal implementation of DLR in the system with high penetration of wind generation under multiple sources of uncertainty. To do so, probabilistic forecasts for DLR are considered, combining with a two-stage stochastic optimization model that cooptimizes the energy production and reserve holding levels in the scheduling stage as well as the re-dispatch actions in the real-time operation stage. Therefore, DLR can be optimally utilized by balancing the benefits of higher utilization of line capacity against the costs of increased holding and utilization of reserve services due to the forecasting error of DLR. Several case studies show that the proposed stochastic framework allows DLR to be optimally utilized, leading to significant operation cost savings and wind curtailment reduction to support the cost-effective integration of wind generation. We also demonstrate that the reserve services can be shared among multiple sources of uncertainty under stochastic dispatch framework, which needs to be recognized in order to avoid the over-estimation of the required reserve services driven by each single source of uncertainty. This paper also identifies the potential synergies between DLR and FACTs devices. The benefits of DLR are shown to increase along with the higher installed capacity of wind generation, but saturate after a certain level of installed capacity.

In the next step, we plan to enhance the modelling framework by explicitly modelling the high-dimensional dependencies among the multiple sources of uncertainty as discussed in [24]. For the long-distance transmission lines, other factors, such as stability constraints and angle limits, may prevent the higher utilization of transfer capability, limiting the benefit of thermal dynamic line rating. We plan to investigate under which conditions and to what extend these factors may potentially affect the utilization of DLR. Furthermore, annual analysis based on European power systems will be carried out with measured system and weather data to further assess the benefits of DLR in supporting high penetration of RES. In particular, the optimal balance between higher utilization of transmission lines and the reduced lifetime need to be investigated. In addition, as there is significant uncertainty associated with the development of renewables, the option value of DLR in supporting costeffective transition toward low carbon system needs to be investigated.

\section{References}

[1] G. Strbac, D. Pudjianto, P. Djapic,and S. Gammons, "Understanding the balancing challenge," Dept. Energy Climate Change, Imperial College London, London, U.K., Aug. 2012.

[2] "The UK renewable energy strategy", Department of Energy \& Climate Change, UK Government, 15 July 2009.

[3] M. W. Davis, "A new thermal rating approach: The real time thermal rating system for strategic overhead conductor transmission lines-Part I: General description and justification of the real time thermal rating system," IEEE Trans. Power Syst., vol.96, no.3, 1977.

[4] IEEE Standard for Calculating the Current-Temperature Relationship for Bare Overhead Conductors, IEEE Std 738-2012, 2012.

[5] "Final Report", TWENTIES project, 2013

[6] C. J. Wallnerstrom, Y. Huang and L. Soder "Impact from Dynamic Line Rating on Wind Power Integration" IEEE Trans. Smart Grid. vol. 6, no. 1, pp. 343-350, 2015.
[7] Tschampion, Michael, Matthias A. Bucher, Andreas Ulbig, and Göran Andersson. " $\mathrm{N}-1$ security assessment incorporating the flexibility offered by dynamic line rating." In PSCC, 2016.

[8] T. Yip, C. An, G. Lloyd, M. Aten and B. Gerri "Dynamic line rating protection for wind farm connections" Proc. CIGRE/IEEE PES Joint Symp. Integration of Wide-Scale Renewable Resources into the Power Delivery Syst., pp. 1-5, Jul., 2009, IEEE.

[9] M.Sara, P.Danny, G.Strbac "Strategic Distribution Network Planning with Smart Grid Technologies" IEEE Trans. Smart Grid., 2016.

[10] K.Kopsidas and A.Kapetanaki "Optimal Demand Response Scheduling with Real Time Thermal Ratings of Overhead Lines for Improved Network Reliability", IEEE Trans. Power Syst., 2016.

[11] M. Nick, O. Alizadeh-Mousavi, R. Cherkaoui and M. Paolone, "Security Constrained Unit Commitment With Dynamic Thermal Line Rating," in IEEE Transactions on Power Systems, vol. 31, no. 3, pp. 2014-2025, May 2016

[12] A. Michiorri, et al., "Forecasting for dynamic line rating", Renewable and Sustainable Energy Reviews, vol. 52, pp. 1713-1730, Dec 2015.

[13] J. L. Aznarte and N. Siebert, "Dynamic Line Rating Using Numerical Weather Predictions and Machine Learning: A Case Study," in IEEE Trans. Power Delivery, vol. 32, no. 1, pp. 335-343, Feb. 2017

[14] Ringelband, T., Schäfer, P. \& Moser, A. Electr Eng (2013) 95: 99. doi:10.1007/s00202-012-0244-8

[15] X Sun, Peter B. Luh, Kwok W. Cheung; W Guan, "Probabilistic forecasting of dynamic line rating for over-head transmission lines", IEEE Power and Energy Society General Meeting, Jul. 2015, pp. 1-5.

[16] F. Qiu and J. Wang, "Distributionally Robust Congestion Management With Dynamic Line Ratings," in IEEE Transactions on Power Systems, vol. 30, no. 4, pp. 2198-2199, July 2015.

[17] MA. Bucher, G. Andersson, "Robust Corrective Control Measures in Power Systems with Dynamic Line Rating", IEEE Transactions on Power Systems 31.3 (2016): 2034

[18] E. M. Constantinescu, V. M. Zavala, M. Rocklin, S. Lee and M Anitescu "A computational framework for uncertainty quantification and stochastic optimization in unit commitment with wind power generation" IEEE Trans. Power Syst., vol. 26, no. 1, pp431-441, 2010.

[19] N. Meinshausen, "Quantile regression forests," The Journal of Machine Learning Research, vol. 7, pp. 983-999, 2006

[20] Kazerooni, A. K., et al. "Dynamic thermal rating application to facilitate wind energy integration." PowerTech, Trondheim. 2011.

[21] Moreno, R., Pudjianto, D., and Strbac, G., "Transmission Network Investment with Probabilistic Security and Corrective Control", IEEE Trans. Power Syst., Vol 28, No 4, pp 3935-3944, Nov 2013.

[22] FICO Xpress Optimization Suite. [Online].

[23] Dupin, Romain, Andrea Michiorri, and Georges Kariniotakis. "Dynamic line rating day-ahead forecasts-cost benefit based selection of the optimal quantile." CIRED Workshop 2016

[24] Wang, Yi, Ning Zhang, Chongqing Kang, Miao Miao, Rui Shi, and Qing Xia. "An efficient approach to power system uncertainty analysis with high-dimensional dependencies." IEEE Trans. Power Syst., 2017

Fei Teng (M'15) received his Bachelor degree from Beihang University, China in 2009 and PhD degree from Imperial College London in 2015. He is currently a lecturer in Control and Power Group at Imperial College London. His research interests include power system control and operation, system flexibility and stochastic optimization.

Romain Dupin is a graduate of Ecole Centrale Lille, where he followed the option Chair of Electrical Networks, and from the University of Lille 1, where he gained a Master's degree in electrical engineering in 2014. Since September 2014, he has been a PhD student in the MINES ParisTech Center for Processes, Renewable Energies and Energy Systems (PERSEE) in Sophia Antipolis, France. He works on developing methods for forecasting dynamic line rating and studies the impacts of dynamic line rating on power system management.

Andrea Michiorri received his Eng. degree in Mechanical Engineering with a specialization in Energy from the University of Rome 'La Sapienza' in 2005. He then obtained a PhD from the University of Durham in 2010 with a dissertation on the thermal state estimation of power system components. $\mathrm{He}$ 
is currently an associate professor at the MINES-ParisTech Center for Processes, Renewable Energies and Energy Systems (PERSEE) in Sophia Antipolis, France, working on the integration of renewable resources and distributed generators into the power system, with a particular focus on the aspects of decision making under uncertainty.

George Kariniotakis (S'95-M'02-SM'11) was born in Athens, Greece. He received his Eng. and M.Sc. degrees from Greece in 1990 and 1992 respectively, and his Ph.D. degree from Ecole des Mines de Paris in 1996. He is currently with the Centre PERSEE of MINES ParisTech as a senior scientist and head of the Renewable Energies and Smartgrids Group. He has authored more than 220 scientific publications in journals and conferences. He has been involved as participant or coordinator in more than $40 \mathrm{R} \& \mathrm{D}$ projects in the fields of renewable energies and distributed generation. Among them, he was the coordinator of some major EU projects in the field of wind power forecasting and integration such as Anemos, Anemos.plus and SafeWind projects. His scientific interests include among others timeseries forecasting, decision making under uncertainty, modelling, management and planning of power systems.

Yanfei Chen received the B.S. degree in electrical engineering from Northumbria University UK in 2011, the M.S. degree in electrical engineering from Imperial College London UK in 2012, and Ph.D. degree from Imperial College London UK in 2017. He is currently a market risk manager at Lloyds Banking Group. His research interests include demand power system operation, FACTS devices and wind energy.

Goran Strbac (M'95) is a Professor of Electrical Energy Systems with Imperial College London. His research interests are in modelling and optimization of electricity system operation and investment, economic and pricing, and integration of new forms of generation and demand technologies. 